\title{
Top-bottom interference effects in Higgs plus jet production at the LHC
}

\author{
Jonas M. Lindert \\ Institute for Particle Physics Phenomenology, Durham University, Durham, DH1 3LE, UK \\ E-mail: jonas.m.lindert@durham.ac.uk
}

Kirill Melnikov*

Institute for Theoretical Particle Physics (TTP), KIT, Karlsruhe, Germany

E-mail: kirill.melnikovakit.edu

\section{Lorenzo Tancredi}

Institute for Theoretical Particle Physics (TTP), KIT, Karlsruhe, Germany

E-mail: lorenzo.tancredi@kit.edu

\section{Chris Wever ${ }^{\dagger}$}

Institute for Theoretical Particle Physics (TTP), KIT, Karlsruhe, Germany \& Institut für Kernphysik (IKP), KIT, 76344 Eggenstein-Leopoldshafen, Germany

E-mail: christopher.weverakit.edu

The next-to-leading order QCD corrections to the top-bottom interference contribution to $H+j$ production at the LHC are presented. The QCD corrections to the interference are large and similar to the QCD corrections to the top-mediated Higgs production cross section. There is also a significant reduction in the mass-renormalization scheme uncertainty once the NLO QCD prediction for the interference is employed.

The European Physical Society Conference on High Energy Physics

5-12 July, 2017

Venice

${ }^{*}$ Supported by the German Federal Ministry for Education and Research (BMBF) under grant 05H15VKCCA.

${ }^{\dagger}$ Speaker. 


\section{Introduction}

Detailed exploration of the Higgs boson properties is a major part of the physics program at the Large Hadron Collider (LHC). It is hoped that studies of the Higgs couplings will reveal possible physics beyond the Standard Model, especially if it mostly manifests itself through interactions with the Higgs bosons. Determination of Higgs couplings at the LHC requires precise theoretical predictions for relevant observables. A case in point is the Higgs boson transverse momentum distribution, whose theoretical understanding is important to properly describe the kinematics of the Higgs decay products, but may also give us access to physics beyond the Standard Model [1]. It was also pointed out in Ref. [2] that studies of the Higgs boson transverse momentum distribution lead to very competitive constraints on the charm and bottom Yukawa couplings.

Higgs bosons at the LHC are mostly produced in gluon collisions through the fluctuation of gluons into quark-antiquark pairs. Because of the differences in fermion Yukawa couplings, the largest contribution to the $g g H$ coupling in the Standard Model comes from top quark loops, followed by bottom and charm loops. For moderate values of the Higgs transverse momentum $p_{\perp} \ll m_{t}$, the top loop contribution can be considered point-like to a very good approximation and its contribution to the transverse momentum distribution is rather advanced, see Refs. [3,4]. However, the bottom and charm loops are not point-like for moderate values of the transverse momentum and their treatment in perturbative QCD is much less understood. To clarify this issue, we report here on the computation of QCD radiative corrections to top-bottom interference contribution to Higgs boson production at the LHC.

\section{Computation of the NLO top-bottom interference contribution}

The calculation of the NLO QCD corrections to the top-bottom interference is non-trivial. The leading order production of the Higgs boson with non-vanishing transverse momentum occurs in different partonic channels, namely $g g \rightarrow H g, q g \rightarrow H q, \bar{q} g \rightarrow H \bar{q}$ and $q \bar{q} \rightarrow H g$. At leading order these processes are mediated by top or bottom loops (the charm contribution in the SM is negligible).

At NLO, the production cross section receives contributions from real and virtual corrections. Since the leading order process only occurs at one-loop, the virtual corrections require two-loop computations that include planar and non-planar box diagrams with internal masses. If we focus on the top-bottom interference and its impact on Higgs production at the LHC, we can simplify the calculation by using the fact that the mass of the $b$-quark, $m_{b} \sim 4.7 \mathrm{GeV}$, is numerically small. Indeed, since $m_{b} \ll m_{H}, p_{\perp}^{\text {typ }}$, where $p_{\perp}^{\text {typ }} \sim 30 \mathrm{GeV}$ is a typical Higgs boson transverse momentum, Feynman diagrams that describe Higgs production can be expanded in series in $m_{b}$ for the purposes of LHC phenomenology. We have checked at leading order that the use of scattering amplitudes either exact or expanded in $m_{b}$ leads to at most few percent differences in the interference contribution to the Higgs $p_{\perp}$ distribution, down to $p_{\perp} \sim 10 \mathrm{GeV}$. We perform the expansion by deriving differential equations for master integrals that are needed to describe the two-loop corrections to $p p \rightarrow H+j$ and then solving them in the limit $m_{b} \rightarrow 0[5,6]$. We note that a similar method was used to compute the top-bottom interference contribution to the inclusive Higgs production cross 
section in Ref. [7]. The two-loop amplitudes mediated by top quark loops, required to describe the interference, are computed in the approximation of an infinitely heavy top quark [8].

To produce physical results for $H+j$ production, we need to combine the virtual corrections discussed above with the real corrections that describe inelastic processes, e.g. $g g \rightarrow H+g g$, $q g \rightarrow H q+g$ etc. The real emission contributions are computed with exact top- and bottom-mass dependence throughout. We use the automated one-loop code OpenLoops [9], that employs a hybrid tree-loop recursion. An important element of the stability of OpenLoops is the employed tensor integral reduction library COLLIER [10]. Its implementation is publicly available [11] and has been applied to compute one-loop QCD and electroweak corrections to multi-leg scattering amplitudes for a variety of complicated processes (see e.g. Refs. [12,13]) and as an input for the real-virtual contributions in NNLO computations (see e.g. Ref. [14]). We have implemented all virtual and real amplitudes in the POWHEG-BOX [15], where infra-red singularities are regularized via FKS subtraction [16]. All OpenLoops amplitudes are accessible via a process-independent interface developed in Ref. [13].

Using the methods described above, we calculated the NLO QCD corrections to the top-bottom interference contribution to $H+j$ production in hadron collisions. We identify the interference contribution through its dependence on top-bottom Yukawa couplings. For the Higgs production cross section, we write

$$
\mathrm{d} \sigma=\mathrm{d} \sigma_{t t}+\mathrm{d} \sigma_{t b}+\mathrm{d} \sigma_{b b}
$$

Individual contributions to the differential cross section scale as $\mathrm{d} \sigma_{t t} \sim \mathscr{O}\left(y_{t}^{2}\right), \mathrm{d} \sigma_{t b} \sim \mathscr{O}\left(y_{t} y_{b}\right)$, $\mathrm{d} \sigma_{b b} \sim \mathscr{O}\left(y_{b}^{2}\right)$. Given the hierarchy of the Yukawa couplings, $y_{t} \sim 1 \gg y_{b} \sim 10^{-2}$, the last term in Eq.(2.1) can be safely neglected. Our main focus is the top-bottom interference contribution $\mathrm{d} \sigma_{t b}$.

\section{Phenomenological results}

In what follows, we present the QCD corrections to the top-bottom interference contribution to the Higgs boson transverse momentum distribution and to the Higgs rapidity distribution in $H+j$ production. We consider proton collisions at the $13 \mathrm{TeV}$ LHC and take the mass of the Higgs boson to be $m_{H}=125 \mathrm{GeV}$. We work within a fixed flavor-number scheme and do not consider bottom quarks as partons in the proton. We use the NNPDF3.0 set of parton distribution functions [17]. We also use the strong coupling constant $\alpha_{s}\left(m_{Z}\right)$ that is provided with this PDF set. We renormalize the $b$-quark mass in the on-shell scheme and use $m_{b}=4.75 \mathrm{GeV}$ as its numerical value. We choose renormalization and factorization scales to be equal and take, as the central value $\mu=H_{T} / 2, \quad H_{T}=\sqrt{m_{H}^{2}+p_{\perp}^{2}}+\sum_{j} p_{\perp, j}$, where the sum runs over all partons in the final state. To quantify the impact of the top-bottom interference on an observable $\mathscr{O}$, it is convenient to define the following quantity

$$
\mathscr{R}_{\text {int }}[\mathscr{O}]=\frac{\int \mathrm{d} \sigma_{t b} \delta(\mathscr{O}-\mathscr{O}(\vec{x}))}{\int \mathrm{d} \sigma_{t t} \delta(\mathscr{O}-\mathscr{O}(\vec{x}))}
$$

where $\vec{x}$ is a set of phase-space variables.

The impact of the top-bottom interference on the Higgs boson transverse momentum distribution is shown in the left plot of Fig. 1. We observe that the leading order interference changes the Higgs boson transverse momentum distribution by $-8 \%$ at $p_{\perp} \sim 20 \mathrm{GeV}$ and $+2 \%$ at $p_{\perp} \sim$ 

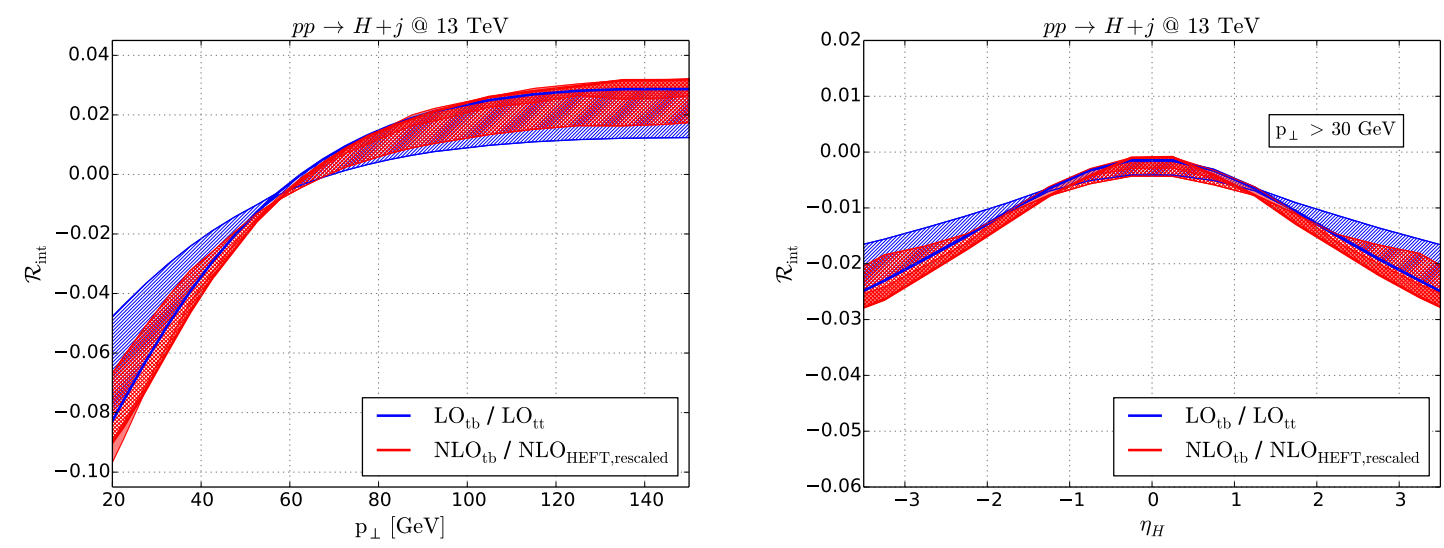

Figure 1: Left: Relative top-bottom interference contribution to the transverse momentum distribution of the Higgs boson at leading (blue) and next-to-leading (red) order in perturbative QCD. At next-to-leading order the interference contribution is shown with respect to the point-like Higgs Effective Field Theory prediction rescaled with exact leading-order top mass dependence. Filled bands, hardly visible at leading order, show the change in $\mathscr{R}_{\text {int }}$ caused by a variation of the renormalization and factorization scales, correlated between numerator and denominator. The hashed bands indicate the uncertainty due to mass-renormalization scheme variation. Right: Relative top-bottom interference contribution to the pseudo-rapidity distribution of the Higgs boson at leading and next-to-leading order in perturbative QCD.

$100 \mathrm{GeV}$. Filled bands in blue for the leading and red for the next-to-leading order predictions show the result for $\mathscr{R}_{\text {int }}\left(p_{\perp}\right)$ computed in the pole mass renormalization scheme. The widths of the bands indicate changes in the predictions caused by variations of renormalization and factorization scales by a factor of two around the central value $\mu=H_{T} / 2$. In fact, we observe that differences between leading and next-to-leading order are very small. For example, $\mathscr{R}_{\text {int }}^{\mathrm{NLO}}\left(p_{\perp}\right)$ appears to be smaller than $\mathscr{R}_{\text {int }}^{\mathrm{LO}}\left(p_{\perp}\right)$ by less than a percent at $p_{\perp}<60 \mathrm{GeV}$ and, practically, coincides with it at higher values of $p_{\perp}$. We emphasise that these small changes in $\mathscr{R}_{\text {int }}$ imply sizable, $\mathscr{O}(40-50 \%)$, corrections to the $t b$ interference proper that, however, appear to be very similar to NLO QCD corrections to the point-like cross section $\sigma_{t t}$. The scale variation bands are very narrow (at leading-order hardly visible) due to a cancellation of large scale variation changes between numerator and denominator in Eq.(3.1). Similar results for the Higgs boson rapidity distribution for events with $p_{\perp}>30 \mathrm{GeV}$ are shown in the right plot of Fig. 1 .

The above result for the scale variation suggests that the uncertainties in predicting the size of top-bottom interference effects in $H+j$ production are small since both the size of corrections and the scale variation bands are similar to the corrections to the point-like $p p \rightarrow H+j$ cross section. Such a conclusion, nevertheless, misses an important source of uncertainties related to a possible choice of a different mass-renormalization scheme. The variation between choosing either the $\overline{\mathrm{MS}}$ scheme and pole mass scheme is shown as hashed bands in Fig. 1, where we have taken $m_{b}=m_{b}^{\text {pole }}$ and $m_{b}=m_{b}^{\overline{\mathrm{MS}}}(100 \mathrm{GeV})=3.07 \mathrm{GeV}$ as the two boundary values. The ambiguity in the leading order value of $\mathscr{R}_{\text {int }}$ is somewhat reduced at next-to-leading order where the effect of the mass renormalization scheme change is less dramatic but, nevertheless significant. For $p_{\perp}<60 \mathrm{GeV}$, the mass renormalization scheme uncertainty is reduced by almost a factor of two, whereas the reduction of uncertainty is only marginal at higher $p_{\perp}$. 


\section{Summary and Outlook}

We computed the NLO QCD corrections to the top-bottom interference contribution to Higgs boson production in association with a jet at the LHC. The corrections to the interference are large yet they appear to track very well corrections to the point-like component of the cross section. The strong dependence of the LO interference on the mass-renormalization scheme is reduced at NLO for moderate values of the Higgs transverse momentum. With this result at hand, one can try to provide the best possible theoretical predictions for the Higgs transverse momentum distribution that combine the known results for the $p_{\perp}$-resummation, NNLO corrections to $H+j$ in the pointlike approximation with the top-bottom interference.

\section{References}

[1] M. Grazzini, A. Ilnicka, M. Spira and M. Wiesemann, arXiv:1612.00283 [hep-ph].

[2] F. Bishara, U. Haisch, P. F. Monni and E. Re, arXiv:1606.09253 [hep-ph].

[3] X. Chen, T. Gehrmann, E. W. N. Glover and M. Jaquier, Phys. Lett. B 740 (2015) 147; R. Boughezal, F. Caola, K. Melnikov, F. Petriello and M. Schulze, Phys. Rev. Lett. 115 (2015) no.8, 082003 ;

F. Caola, K. Melnikov and M. Schulze, Phys. Rev. D 92 (2015) no.7, 074032; X. Chen,

J. Cruz-Martinez, T. Gehrmann, E. W. N. Glover and M. Jaquier, JHEP 1610 (2016) 066;

R. Boughezal, C. Focke, W. Giele, X. Liu and F. Petriello, Phys. Lett. B 748 (2015) 5.

[4] P. F. Monni, E. Re and P. Torrielli, Phys. Rev. Lett. 116, no. 24, 242001 (2016).

[5] K. Melnikov, L. Tancredi and C. Wever, JHEP 1611, 104 (2016).

[6] K. Melnikov, L. Tancredi and C. Wever, arXiv:1702.00426 [hep-ph].

[7] R. Mueller and D. G. Öztürk, JHEP 1608, 055 (2016).

[8] C. R. Schmidt, Phys. Lett. B 413, 391 (1997).

[9] F. Cascioli, P. Maierhöfer and S. Pozzorini, Phys. Rev. Lett. 108, 111601 (2012).

[10] A. Denner, S. Dittmaier and L. Hofer, Comput. Phys. Commun. 212, 220 (2017); A. Denner and S. Dittmaier, Nucl. Phys. B 658, 175 (2003); A. Denner and S. Dittmaier, Nucl. Phys. B 734, 62 (2006); A. Denner and S. Dittmaier, Nucl. Phys. B 844, 199 (2011).

[11] The OpenLoops one-loop generator by F. Cascioli, J. Lindert, P. Maierhöfer and S. Pozzorini is publicly available at http://openloops. hepforge.org.

[12] S. Kallweit, J. M. Lindert, P. Maierhöfer, S. Pozzorini and M. Schönherr, JHEP 1604, 021 (2016); S. Höche, P. Maierhöfer, N. Moretti, S. Pozzorini and F. Siegert, arXiv:1607.06934 [hep-ph].

[13] T. Jezo, J. M. Lindert, P. Nason, C. Oleari and S. Pozzorini, Eur. Phys. J. C 76, no. 12, 691 (2016).

[14] F. Cascioli et al., Phys. Lett. B 735, 311 (2014); D. de Florian, M. Grazzini, C. Hanga, S. Kallweit, J. M. Lindert, P. Maierhöfer, J. Mazzitelli and D. Rathlev, JHEP 1609, 151 (2016); M. Grazzini, S. Kallweit, S. Pozzorini, D. Rathlev and M. Wiesemann, JHEP 1608, 140 (2016)

[15] P. Nason, JHEP 0411, 040 (2004); S. Frixione, P. Nason and C. Oleari, JHEP 0711, 070 (2007); S. Alioli, P. Nason, C. Oleari and E. Re, JHEP 1006, 043 (2010); T. Jezo and P. Nason, JHEP 1512, 065 (2015).

[16] S. Frixione, Z. Kunszt and A. Signer, Nucl. Phys. B 467, 399 (1996).

[17] R. D. Ball et al. [NNPDF Collaboration], JHEP 1504, 040 (2015). 